\title{
HIV-1 protease flaps spontaneously close to the correct structure in simulations following manual placement of an inhibitor into the open state
}

\author{
Viktor Hornak $^{\%}$, Asim Okur ${ }^{\dagger}$, Robert C. Rizzo \#, , and Carlos Simmerling ${ }^{\dagger}, \%,{ }^{*}$ \\ $\dagger$ Department of Chemistry, Stony Brook University, Stony Brook, NY 11794 \\ \# Department of Applied Mathematics and Statistics, Stony Brook University, Stony Brook, NY 11794 \\ \% Center for Structural Biology, Stony Brook University, Stony Brook, NY 11794 \\ ${ }^{*}$ Computational Science Center, Brookhaven National Laboratory, Upton NY11973
}

\begin{abstract}

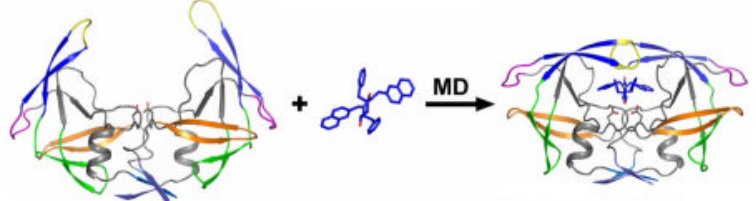

We report unrestrained, all-atom molecular dynamics simulations of HIV-1 protease (HIV-PR) with a continuum solvent model that reproducibly sample closing of the active site flaps following manual placement of a cyclic urea inhibitor into the substrate binding site of the open protease. The open form was obtained from the unbound, semi-open HIV-PR crystal structure, which we recently reported ${ }^{1}$ to have spontaneously opened during unrestrained dynamics. In those simulations, the transiently open flaps always returned to the semi-open form that is observed in all crystal structures of the free protease. Here, we show that manual docking of the inhibitor reproducibly induces spontaneous conversion to the closed form as seen in all inhibitor-bound HIV-PR crystal structures. These simulations reproduced not only the greater degree of flap closure, but also the striking difference in flap "handedness" between bound and free enzyme (Figure 1). In most of the simulations, the final structures were highly accurate. Root mean square deviations (RMSD) from the crystal structure of the complex were $\sim 1.5 \AA$ (averaged over the last 100ps) for the inhibitor and each flap despite initial RMSD of $2-5 \AA$ for the inhibitors and $6-11 \AA$ for the flaps. Key hydrogen bonds were formed between the flap tips and between flaps and inhibitor that match those seen in the crystal structure. The results demonstrate that all-atom simulations have the ability to significantly improve poorly docked ligand conformations and reproduce large-scale receptor conformational changes that occur upon binding.
\end{abstract}

We report unrestrained, all-atom molecular dynamics simulations of HIV-1 protease (HIVPR) with a continuum solvent model that reproducibly sample closing of the active site flaps following manual placement of a cyclic urea inhibitor into the substrate binding site of the open protease. The open form was obtained from the unbound, semi-open HIV-PR crystal structure, which we recently reported ${ }^{1}$ to have spontaneously opened during unrestrained dynamics. In those simulations, the transiently open flaps always returned to the semi-open form that is

E-mail: carlos.simmerling@stonybrook.edu. 
observed in all crystal structures of the free protease. Here, we show that manual docking of the inhibitor reproducibly induces spontaneous conversion to the closed form as seen in all inhibitor-bound HIV-PR crystal structures. These simulations reproduced not only the greater degree of flap closure, but also the striking difference in flap "handedness" between bound and free HIV-PR (Figure 1). In most of the simulations, the final structures were highly accurate. Root mean square deviations (RMSD) from the crystal structure of the complex were $\sim 1.5 \AA$ (averaged over the last 100ps) for the inhibitor and each flap despite initial RMSD of $2-5 \AA$ for the inhibitors and 6-11 $\AA$ for the flaps. Key hydrogen bonds were formed between the flap tips and between flaps and inhibitor that match those seen in the crystal structure. The results demonstrate that all-atom simulations have the ability to significantly improve poorly docked ligand conformations and reproduce large-scale receptor conformational changes that occur upon binding.

Due to its central role in processing viral polypeptide precursors, HIV-PR continues to be one of the primary targets of anti-AIDS drug discovery. A greater understanding of the mechanistic events associated with HIV-PR binding is critical for the design of more potent and novel inhibitors of this viral enzyme. An extensive set of X-ray crystal structures of HIV-1 protease has been solved, revealing a $\mathrm{C} 2$ symmetric homodimer with a large substrate binding pocket covered by two glycine rich $\beta$-hairpins, or flaps ${ }^{2,3}$. Consistent structural differences are present between the bound and free states of the protein (Figure 1). In all of the inhibitor-bound forms, the flaps are pulled in towards the bottom of the active site (the "closed" form), while the structures for the unbound protease all adopt a "semi-open" conformation with the flaps shifted away from the dual Asp25-Thr26-Gly27 catalytic triads, but still substantially closed over the active site and in contact with each other. A more striking difference is that the relative orientation (the "handedness") of the $\beta$-hairpin flaps is reversed in the two forms (Figure 1).

We recently reported 1 the first simulations that sampled spontaneous opening of unbound HIVPR with subsequent return to the crystallographic semi-open form. The closed inhibitor-bound HIV-PR was stable under the same conditions. In the present study we employ the same Amber simulation protocol and parameters, including a modified ${ }^{4}$ Generalized Born 5 implicit water model and no cutoff on nonbonded interactions. We simulated the wild type sequence in complex with the cyclic urea inhibitor XK263 (pdb code 1HVR) ${ }^{7}$. Consistent with experiments on cyclic urea-bound HIV-PR, both catalytic Asp side chains were protonated 6 . Flap RMSDs were calculated for backbone of residues $46-55$ or $46^{\prime}-55^{\prime}$. Inhibitor RMSDs used all atoms. All RMSD values were calculated after a best-fit to the non-flaps backbone of HIV-PR (residues 6-38 and 55-94 in each monomer, excluding the termini and flexible elbow regions). Reported final RMSD values reflect averages obtained over the last 100ps.

We generated two initial structures, using open conformations with flap RMSD values of 6 to $11 \AA$. The inhibitor was manually docked into both, resulting in inhibitor RMSD values of 2.4 $\AA$ and $5.3 \AA$, with the second intended only to place the inhibitor roughly in the binding site cavity. We note that since the open structures were obtained from a simulation initiated with the unbound, semi-open crystal structure, no "memory" of the bound HIV-PR conformation could have been present. Importantly, the same open conformations returned to the semi-open form in simulations without inhibitor ${ }^{1}$.

With the more accurately docked inhibitor (2.4 $\AA$ RMSD), the flaps spontaneously closed over the inhibitor after only $\sim 50$ ps of MD at $310 \mathrm{~K}$ (Figure 2), reaching a plateau at $\sim 4 \AA$ RMSD. During the flap closing, the RMSD of the inhibitor rose above $6 \AA$, moving significantly from the initial docked position. This change reflected a shift of the inhibitor inside the open binding site and formation of multiple contacts with one of the flaps, including a hydrogen bond between the urea carbonyl oxygen and that flap tip Ile50 amide hydrogen, as seen in the crystal structure of this complex. At $\sim 200 \mathrm{ps}$, the flaps closed further and the inhibitor shifted in the 
binding site to add a hydrogen bond between the urea carbonyl and the other flap tip (Ile50'). At this point the inhibitor RMSD was reduced from $\sim 4.5 \AA$ to $\sim 1.5 \AA$ (Figure S1) and the flaps adopted the correct conformation (average RMSDs of $\sim 1.5 \AA$ and $1.9 \AA$, sampling values as low as $0.9 \AA$ ). An inter-flap hydrogen bond formed between the amides of Gly51 (donor) and Ile50' (acceptor), also seen in the crystal structure of this complex. These average final RMSD values are similar to what we recently reported ${ }^{1}$ during $28 \mathrm{~ns}$ stable simulations of the complex when initiated from the bound crystal structure. This simulation was continued to a total time of $2 \mathrm{~ns}$ with no significant change. Two additional simulations were performed from the same initial structure, with the flaps again adopting (at $600 \mathrm{ps}$ and $2 \mathrm{~ns}$ ) the correct closed handedness and average $\sim 1.5 \AA$ RMSD for the inhibitor and both flaps.

Three simulations from the less accurately docked inhibitor (initially $5.3 \AA$ ) were performed at $300 \mathrm{~K}$. In the first, the flaps RMSD fell below $2 \AA$ at $\sim 1 \mathrm{~ns}$ as the flaps adopted the closed handedness. During the same time, the ligand located the correct binding site and its RMSD was reduced from $\sim 6 \AA$ to $\sim 2 \AA$. In this simulation, the flap RMSDs remained above $2 \AA$ for $\sim 5 \mathrm{~ns}$ before finally reaching the accurate (average $\sim 1.5-1.6 \AA$ ) conformation that was adopted more rapidly in the simulations described above. Further analysis revealed that the final conformation of the inhibitor in this simulation was not identical to that seen in the crystal structure. A rotation of one of the inhibitor's $\mathrm{P} 2 / \mathrm{P} 2^{\prime}$ naphthyl substituents by $180^{\circ}$ during dynamics resulted in a slight shift of the inhibitor in the binding pocket to accommodate the resulting asymmetry (Figure S2). The closed flaps prevented further rotation, and the final inhibitor RMSD value at $14 \mathrm{~ns}$ was $\sim 2 \AA$, compared to the $1.5 \AA$ values described above. Nevertheless, the final structure adopted by the complex remained highly similar to that seen in the crystal (Figure 3), particularly given the initial inhibitor RMSD value of $5.3 \AA$.

Two of the simulations differed from the others in that the flaps initially closed to the handedness observed in the unbound, semi-open crystal structures, similar to what we reported for the closing in simulations of unbound HIV-PR. This flap configuration was unstable in both simulations; one subsequently converted to the correct closed form after $\sim 5 \mathrm{~ns}$, where it remained for the duration of the $\sim 40 \mathrm{~ns}$ simulation. The other re-opened and did not close properly during the remaining 40ns. This was the only simulation of the six that did not convert to the correct structure. In contrast, simulations of inhibitor docked to the semi-open crystal structure did not convert to correct closed structure on this timescale. These will be discussed elsewhere.

Taken together, these results provide compelling evidence that the large-scale flap opening events that we recently reported are directly relevant to inhibitor binding. The results also suggest that the inhibitor may play a role in determining the structure adopted by the closing HIV-PR flaps. Five out of six fully unrestrained simulations with inhibitors docked into an open state obtained from the unbound, semi-open crystal structure spontaneously underwent large conformational changes and adopted the closed form. Closure was accompanied by correct reversal of flap handedness and formation of all key hydrogen bonds between the flap tips and between flaps and the inhibitor that are present in the crystal structure (Figure S3).

Our ability to sample these events on an affordable timescale was facilitated by the use of a low-viscosity implicit water model. Future studies using potentially more accurate explicit solvent models would be highly desirable. These would not only facilitate quantitative comparison of timescales but also provide useful insight into a possible structural role for water during dynamic binding events. For example, a well-ordered water molecule (WAT301) mediating interaction between the flap tips and peptide-based inhibitors is observed in multiple HIV-PR crystal structures ${ }^{3,7}$. This water molecule is not present in the complex with the cyclic urea inhibitor that we employed, thus an explicit water model may be needed to obtain the same high level of accuracy with other inhibitors. 


\section{Supplementary Material}

Refer to Web version on PubMed Central for supplementary material.

\section{Acknowledgements}

CS thanks Adrian Roitberg and Dan Raleigh for discussions and Roberto Gomperts for important Amber optimizations and Altix resources from the SGI Engineering group. Financial support from NIH (GM6167803) and DOE (DEAC02-98CH10886) and supercomputer time at NCSA (NPACI MCA02N028) are gratefully acknowledged. C.S. is a Cottrell Scholar of Research Corporation.

\section{References}

1. Hornak V, Okur A, Rizzo RC, Simmerling C. Proceedings of the National Academy of Sciences of the United States of America 2006;103:915-920. [PubMed: 16418268]

2. Vondrasek J, Wlodawer A. Proteins-Structure Function and Genetics 2002;49:429-431.

3. Wlodawer A, Vondrasek J. Annual Review of Biophysics and Biomolecular Structure 1998;27:249_ 284.

4. Onufriev A, Bashford D, Case DA. Journal of Physical Chemistry B 2000;104:3712-3720.

5. Still WC, Tempczyk A, Hawley RC, Hendrickson T. Journal of the American Chemical Society 1990;112:6127-6129.

6. Yamazaki T, Nicholson LK, Torchia DA, Wingfield P, Stahl SJ, Kaufman JD, Eyermann CJ, Hodge CN, Lam PYS, Ru Y, Jadhav PK, Chang CH, Weber PC. Journal of the American Chemical Society 1994;116:10791-10792.

7. Lam PYS, Jadhav PK, Eyermann CJ, Hodge CN, Ru Y, Bacheler LT, Meek JL, Otto MJ, Rayner MM, Wong YN, Chang CH, Weber PC, Jackson DA, Sharpe TR, Ericksonviitanen S. Science 1994;263:380-384. [PubMed: 8278812]

8. Spinelli S, Liu QZ, Alzari PM, Hirel PH, Poljak RJ. Biochimie 1991;73:1391-1396. [PubMed: 1799632] 


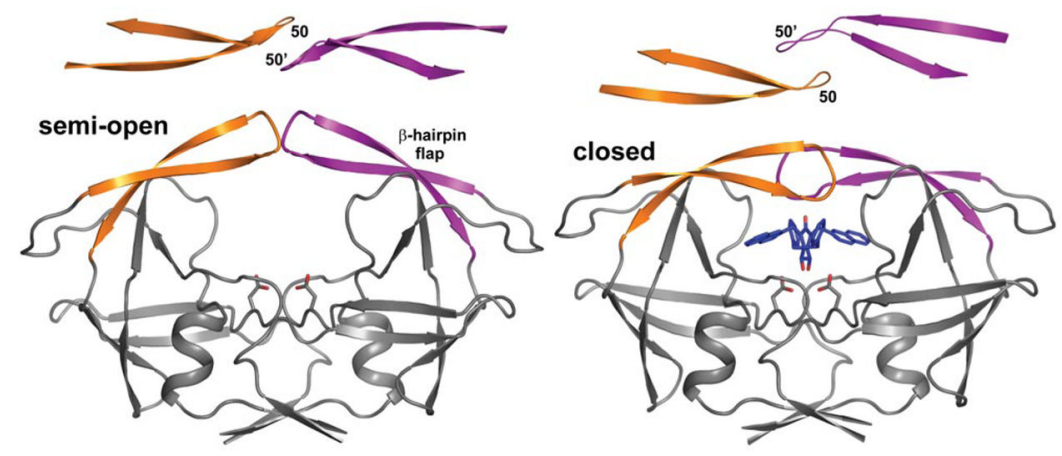

Figure 1.

Crystal structures of HIV-PR: free (left, pdb $1 \mathrm{HHP}^{8}$ ) and with bound inhibitor (right, $1 \mathrm{HVR}^{7}$ ). A top view of the flaps is shown to illustrate the change in handedness that occurs upon inhibitor binding. 


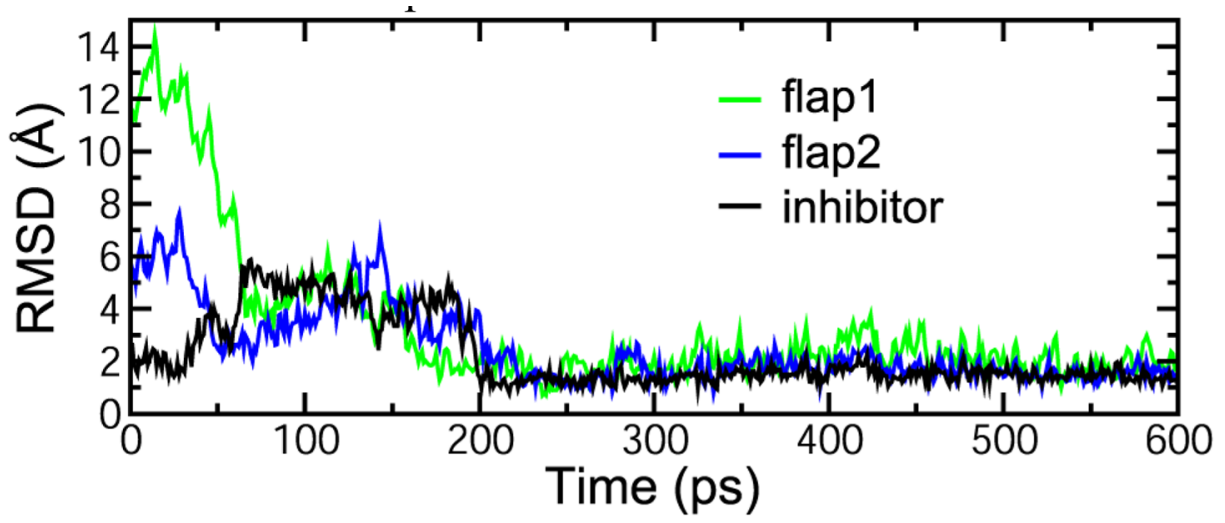

Figure 2.

RMSD values during MD simulation following docking the inhibitor into fully open HIV-PR. The inhibitor (all atoms) and each flap (backbone) are compared to the bound, closed crystal structure of the complex, best-fit to the non-flaps portion of the protease. Final values fluctuate

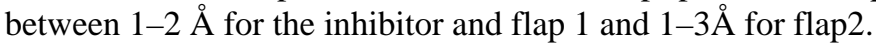




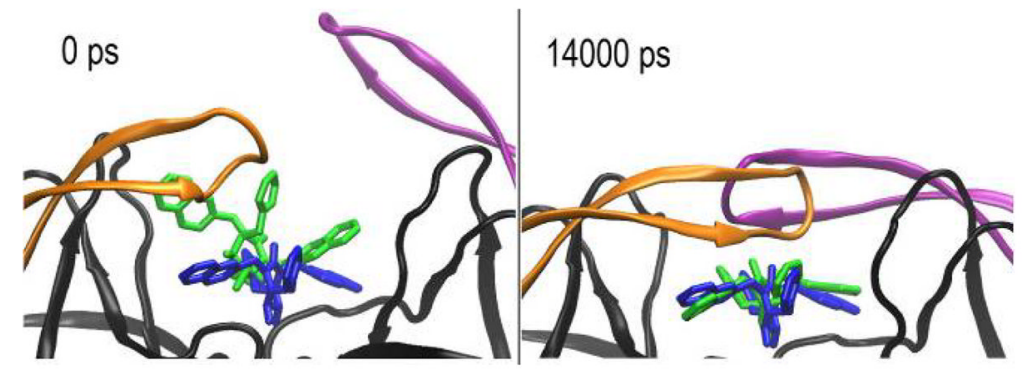

Figure 3.

Simulated HIV-PR binding site and inhibitor after rough manual docking of the inhibitor into the open state (left) and after MD refinement (right). The crystallographic position of the inhibitor is shown in blue. 